\title{
(息)
}

Citation:

Richardson, N (2018) Corporate social responsibility or sustainability in music festivals. International Journal of Organizational Analysis. ISSN 1055-3185 DOI: https://doi.org/10.1108/IJOA-03-20181368

Link to Leeds Beckett Repository record:

https://eprints.leedsbeckett.ac.uk/id/eprint/5583/

Document Version:

Article (Accepted Version)

The aim of the Leeds Beckett Repository is to provide open access to our research, as required by funder policies and permitted by publishers and copyright law.

The Leeds Beckett repository holds a wide range of publications, each of which has been checked for copyright and the relevant embargo period has been applied by the Research Services team.

We operate on a standard take-down policy. If you are the author or publisher of an output and you would like it removed from the repository, please contact us and we will investigate on a case-by-case basis.

Each thesis in the repository has been cleared where necessary by the author for third party copyright. If you would like a thesis to be removed from the repository or believe there is an issue with copyright, please contact us on openaccess@leedsbeckett.ac.uk and we will investigate on a case-by-case basis. 


\title{
TITLE
}

\section{Corporate Social Responsibility or Sustainability in music festivals?}

\begin{abstract}
Purpose - To establish whether social considerations are valued within UK music festivals.

Methodology - A case study featuring 21 semi-structured interviews with stakeholders delivering 7 music festivals. Thematic analysis enabled identification of insights into differences between organisers and suppliers.
\end{abstract}

Findings - The respondents were positive towards sustainability however CSR had little recognition. Both stakeholder groups adopted 'ethical' practices. Suppliers want organisers to be transparent and fair. Organisers want supplier to comply with their approaches. All stakeholders need to improve their communications.

Practical implications - Festivals operate in increasingly competitive environments hence the insights herein should improve stakeholder and festivalgoer engagement.

Originality - Few studies of the adoption of social considerations exist within the creative industries.

Research limitations - As a small qualitative study, it is not representative of the sector. Furthermore suppliers may be unwilling to critique festivals. Social desirability bias may be evident.

Key words - Sustainability, CSR, Music Festivals, Stakeholders, Suppliers, Perceptions, Awareness

Paper type - Research 


\section{INTRODUCTION}

A long-standing discourse exists apropos the relationship between business and society (Garriga \& Mele, 2004). Social considerations are frequently given less attention than economic and environmental concerns (Musgrave \& Raj, 2009). This paper seeks to address this by investigating social considerations, namely Corporate Social Responsibility (CSR) and the social aspects of Sustainable Development (SD), within small UK music festivals. Whilst larger festivals have established policies, independent festivals substantially outnumber their larger peers. Indeed they are the bellwether of UK music festivals.

As leaders influence responsible behaviour (Andersen, 2017), this paper contrasts the 'social' attitudes and motivations of festival organisers (and employees) with their suppliers. Festivals are unlike other services as they involve high degrees of consumer engagement, often in areas of outstanding natural beauty. Festivalgoers not only consume but also cocreate the festival. The emergence of consumers as co-creators of creative products has stimulated much cultural interaction and interchange, which has been harnessed by firms in the creative industries (UNCTAD, 2008). Hence, stakeholders must align their values with changing consumer behaviour e.g. increasingly ethical and environmental spending patterns (Henderson \& Musgrave, 2014). Whilst festivals are increasingly being ecologically audited, little research on the social considerations exists. Indeed festivals have largely been ignored in fields such as sociology, anthropology, strategy and management (Moeran \& Strandgaard-Pedersen, 2011).

Festivals rarely last more than a few days and this ephemerality shapes the relationships between organisers and suppliers. Both groups are predominantly sole traders, microenterprises or small-to-medium size enterprises (SMEs). SMEs represent over $99 \%$ of enterprises in the UK (Moore \& Manring, 2009) and have significant environmental impacts being responsible for up to $70 \%$ of UK industrial pollution (Walker \& Preuss, 2008). They have differing aims and objectives e.g. being profit-making, not-for-profit or community based. Hence the relationship (between organisers and the diverse range of suppliers) is unique. Therefore, the first research objective $\left(\mathrm{RO}_{1}\right)$ is

"To ascertain how both stakeholder groups perceive social practices".

To test $\mathrm{RO}_{1}$, the awareness and extent to which social considerations resonate with organisers and suppliers is investigated. This evaluates whether CSR and/or SD are frequently used and have attracted broader awareness (Barkemeyer et al, 2009).

It is useful to conceptualize the adoption of social practices from the perspectives of organisers and suppliers. This necessitates a second research objective $\left(\mathrm{RO}_{2}\right)$, which is 
"To identify if patterns exist between the stakeholder groups apropos adopting CSR or SD".

To test $\mathrm{RO}_{2}$ this paper identifies the adoption of social practices and issues that arise. This extends the work of Haberberg et al (2010), who found patterns existed in CSR adoption across organisations, and Getz (2010) who studied festivals and argued the need for social considerations therein.

Divergences in the adoption of social practices, could impact on the delivery of festivals to increasingly discerning festivalgoers. It is prudent to consider what steps could be taken to mitigate any divergences. Therefore $\left(\mathrm{RO}_{3}\right)$ is

"to suggest practices that could address divergences between the stakeholder groups apropos adopting CSR or SD".

This contributes to studies where standards were viewed with suspicion (Oates et al, 2008). Furthermore as festivals are ephemeral, this objective questions whether compliance (with CSR and/or SD standards) should be mandatory and enforced by organisers (Musgrave \& Raj, 2009).

\section{LITERATURE REVIEW}

As $\mathrm{RO}_{1}$ alludes to perception it is useful to first reflect on the social considerations themselves.

\section{CSR}

If ethics has any meaning

"it will be because we need to justify our lives to ourselves and to others ... (and if) ... business is on one side and ethics is on the other, then we'll have a gap that may come to be known as "corporate social responsibility" (Freeman, 2008, p164).

The notion of companies acting with society's interests at heart is not new, indeed social considerations for businesses were discussed in the 1920s (Windsor, 2001). Previous studies found correlations between profitability and CSR measures such as employee and community relations (Garriga \& Mele, 2004). Whilst such correlations are difficult to measure, festivals have a long tradition of making contributions to social causes (see http://www.glastonburyfestivals.co.uk).

CSR is complex as it draws on economics, politics, social integration and ethics (Garriga \& Mele, 2004). It is a broad, eclectic field with loose boundaries, multiple memberships, 
different perspectives and interdisciplinary (Carroll, 1991). CSR can be seen as a construct that is individual to stakeholders and has been referred to as the social contract organisations have with their stakeholders (Jones et al, 2009). This social contract can provide benefits for organisations e.g. it offers companies an opportunity to mitigate negative consequences of their operations through image management (Wood, 2008).

$\mathrm{RO}_{2}$ is necessary as patterns exist in CSR adoption across organisations, with stakeholders exhibiting varying degrees of commitment (Haberberg et al, 2010). Suppliers comply for differing reasons e.g. being enlightened, coerced or mimetic. Customers can be the forgotten stakeholders (Belz \&Peattie, 2012) as organisations are not necessarily consistent in their approach. Furthermore, conflicting conceptions of a festival's role and purpose may also be reflected in how the relevance of CSR is evaluated (Margolis \& Walsh, 2003).

Carroll and Wood are widely cited as having played key CSR roles having contributed to identifying he different levels at which organisations address social considerations (Windsor, 2001). Carroll's pyramid (Fig 1) is intended to portray how the total CSR of business comprises distinct components that taken together constitute the whole (Carroll, 1991).

\begin{tabular}{|l|l|}
\hline \multicolumn{2}{|c|}{ Fig 1 Carroll's CSR Pyramid portraying levels of organisational CSR } \\
\hline Discretionary level & $\begin{array}{l}\text { Organisation goes beyond stakeholder views of everyday expected } \\
\text { duty, what is just and fair, often involving philanthropy, and is an } \\
\text { exemplary corporate citizen. }\end{array}$ \\
\hline Ethical level & $\begin{array}{l}\text { Organisation views its responsibility to satisfy society's expectations } \\
\text { to go beyond basic legal requirements and do what is just and fair, } \\
\text { and their practice reflects this. Focused on business ethics in a wide } \\
\text { stakeholder context }\end{array}$ \\
\hline Legal level & $\begin{array}{l}\text { Organisation obeys all the laws and rules applied by the state. (E.g. } \\
\text { tax, regulation, etc.) Concerned as with the law and legal rights, } \\
\text { duties, rules and obligations are built }\end{array}$ \\
\hline Economic level & $\begin{array}{l}\text { Organisation produces products and services that society wants and } \\
\text { sells them at a profit. Base starting point the economy and economic } \\
\text { performance. This is seen as pivotal }\end{array}$ \\
\hline \multicolumn{2}{|c|}{ Source adapted from Carroll (1979, 1991) \& Wood (1991) as cited in Jones et al (2009a, p302) } \\
\hline
\end{tabular}

Jones et al (2009, pp302-303) cite a number of issues with Carroll's Pyramid namely

- the levels are not mutually exclusive

- it is a staged hierarchy where movement results from fixed criteria

- the dynamism of the social, economic and business world is only partially captured

- it is a theoretical abstract removed from the complex realities of the world it seeks to explain

Increasingly scholars criticise the 'CSR business case' as it assumes CSR is central to organisational culture and beliefs whereas it may simply be an afterthought (Jones et al, 2009). It often concerns the enhancement of a company's competitive advantage, corporate reputation, resource efficiency, impact on staff morale and being portrayed as a proxy for 
competent management (Barkemeyer, 2009). Rather than accepting CSR at face value doubts are raised regarding the general suitability of the current mode of predominantly voluntary, business-led CSR instruments to tackle some of the most pressing developmental challenges (ibid).

In order to mitigate divergences (see $\mathrm{RO}_{3}$ ) between the stakeholder groups, organisers must assume suppliers will comply with the festival's policies. Organisers who are highly motivated may adopt idealistic CSR stances or even ones of enlightened self-interest whereas stakeholders on whom they rely may only do so when coerced. This poses risks for motivated organisers who face disadvantages when pursuing 'costly' sustainable actions as such costs may not be borne by competitors (Pacheco et al, 2010).

Although CSR predates the SD movement (Emery, 2012), the recent inclusion of ecological matters in CSR (see the CSI-CSR continuum proposed by Jones et al, 2009) suggests it fails to provide a complete answer to the issues raised by the sustainability agenda.

\section{$S D$}

In testing $\mathrm{RO}_{1}$ is worth noting that many $\mathrm{SD}$ definitions exists which itself can create barriers to adoption (Barkemeyer, 2009). In 1997 Hart's 'Beyond Greening' brought SD to the wider business community. Concurrently, the Brundtland Commission report defined SD as 'development that meets the needs of the present without compromising the ability of future generations to meet their own needs' (Emery, 2012, p20). Soon after, Elkington offered the 'Triple-Bottom-Line' (TBL) where the traditional economic focus is complemented by the foci of societal and environmental responsibility (Elkington, 1998). TBL, often paraphrased as 'People-Planet-Profit' can be described as an enduring, balanced approach to economic activity, environmental responsibility and social progress (ibid).

The TBL is increasingly being accepted (Emery, 2012) however many social problems remain stubbornly intractable (Belz \& Peattie, 2012). This was supported by Getz (2007, 2010) who investigated the economic impact of events. Tellingly, he stressed that the impacts of events should include the community and the environment. Despite Elkington's assertion that TBL 'took off' around the turn of the century (Elkington, 2004), it has not yet attracted broader public awareness (Barkemeyer et al, 2009). A lack awareness does not necessarily mean TBL has lacked impact within organisations, it could however point to poor communications. If the success of social programmes depends on broader awareness and participation (ibid), investigating $\mathrm{RO}_{2}$ may ascertain whether terminological confusion can contribute to divergence between the stakeholder groups. 
Many forces underscore the emerging opportunities for festivals to proactively adopt social practices including extended, interconnected supply chains and rapidly changing markets (Moore \& Manring, 2009). Adopting standards for sustainable event management, namely ISO 20121:2012, could help. This specifies requirements for event sustainability management systems and provides guidance on conformance (ISO, 2015). It is useful to ascertain awareness of such standards and whether adoption could address divergence between the stakeholder groups $\left(\mathrm{RO}_{3}\right)$.

\section{RESEARCH METHODOLOGY}

Getz (2010) identified issues with the scale of 'festival' research. He used 'festivals' as the key search-term and generated a response that is too large to be useful (Fig 2). Herein the search-term was amended to 'music festivals', however the results were still arguably too large

\begin{tabular}{|c|c|c|c|c|c|}
\hline \multicolumn{6}{|c|}{ Fig 2 Comparing search results for 'music festival' } \\
\hline & Google & Google Scholar & \multicolumn{3}{|c|}{ Academic databases } \\
\hline Feb 2009 & 284,000 & 327,000 & & & 506 \\
\hline \multirow[t]{8}{*}{ Jan 2015} & $255,000,000$ & 471,000 & Ebsco & 30243 & \\
\hline & & & Emerald & 1096 & \\
\hline & & & Jstor & 2027 & \\
\hline & & & Mintel & 250 & \\
\hline & & & Warc & 489 & \\
\hline & & & Scopus & 368 & \\
\hline & & & Web of Science & 229 & \\
\hline & & & Total & 34702 & \\
\hline \multicolumn{6}{|c|}{$\begin{array}{l}\text { Source: Feb } 2009 \text { data from Getz (2010) using 'festivals'; Jan } 2015 \text { data using } \\
\text { 'music festival' with Ebsco, Emerald, JSTOR, Mintel, World of Science, Scopus } \\
\text { and WARC databases }\end{array}$} \\
\hline
\end{tabular}

Whilst the Google Scholar findings portrayed a $44 \%$ increase over the 6 -year period, the standout figures are the dramatic increase in Google results and in databases. Getz's 2009 figure of 506 should be viewed with some scepticism when compared to the Google Scholar figure of 327,000 results. These naturally include results germane to research beyond the remit of this study.

\section{The UK Music Festival Sector}

Music festivals annually generate thousands of unique days of entertainment for millions of festivalgoers (Mintel, 2017). In 2012 the attendance at festivals rose to $12 \%$ of the UK population having visited a festival in the year (Mintel, 2012). Britain hosted 715 music festivals in 2010, providing 2,820 days' entertainment (Fig 3). 


\begin{tabular}{|l|c|c|c|c|c|}
\hline \multicolumn{5}{|c|}{ Fig 3 Number of } \\
$\mathbf{2 0 1 0}$ music festivals and days in the UK by region \\
(Source - adapted from Mintel, 2010)
\end{tabular}

These figures, whilst impressive, do not consider the multiplier effect of festivals, namely artists anticipate increasing in sales of music and merchandise by playing festivals. Whilst Fig 3 recognises the quantity of festivals it does not differentiate between different types of festival.

\section{A Music Festival Typology}

Festivals can be viewed as complex periodic events creating demand in specific times and places (Andersson \& Getz, 2009). Festivals are temporarily bounded in terms of both duration and regularity (Moeran \& Strandgaard-Pedersen, 2011). Some are 'one-offs' for example the Jubilee in 2012 featured free music festivals (Andersson \& Getz, 2009) whilst others last longer. Clearly the nature of the festival can shape the attitudes towards social considerations. However, no widely acceptable typology has emerged (Getz, 2010; Maeng et al, 2016). A festival typology (Fig 4) is necessary as this paper is predicated on research undertaken across a range of festivals. 


\begin{tabular}{|c|c|c|}
\hline \multicolumn{3}{|c|}{ Fig 4 A typology of music festivals } \\
\hline Indicator & Value & Factors \\
\hline Frequency & One-off, recurring, irregular & Annual, Biannual, Ad-hoc \\
\hline Scope & $\begin{array}{l}\text { Visitors, Linked to other events e.g. } \\
\text { Jubilee or Olympic Games }\end{array}$ & $\begin{array}{l}\text { Local, Regional, National, } \\
\text { International }\end{array}$ \\
\hline Established & $\begin{array}{l}\text { One-off, New, Nascent }(<5 \text { yrs old) } \\
\text { Well established (over } 5 \text { years old) }\end{array}$ & \\
\hline Scale & $\begin{array}{l}\text { City wide, mega, regional, boutique } \\
\text { Community, small scale, }\end{array}$ & $\begin{array}{l}\text { Small }(<5000 \text { festivalgoers), } \\
\text { Medium }(5000-9999), \text { large } \\
(10000-30000), \text { Mega }(>30000)\end{array}$ \\
\hline Type & $\begin{array}{l}\text { Special, genre, social, major, } \\
\text { hallmark, organizing body, audience } \\
\text { specific, permanent site, } \\
\text { promotional campaign }\end{array}$ & \\
\hline Destination & $\begin{array}{l}\text { Single site, multi-site, fixed, mobile, } \\
\text { urban, rural, 'rurban' (e.g. } \\
\text { racecourse), remote, coastal }\end{array}$ & $\begin{array}{l}\text { Domestic } \\
\text { International }\end{array}$ \\
\hline Sector & $\begin{array}{l}\text { Private, Public, Not-for-profit, } \\
\text { Government, Hybrids }\end{array}$ & $\begin{array}{l}\text { Single organiser, Partnerships, } \\
\text { Joint ventures }\end{array}$ \\
\hline \multicolumn{3}{|c|}{$\begin{array}{c}\text { Source - adapted from Andersson \& Getz (2009), Amit \& Zott (2001), Bird (2007), Collins (2010) } \\
\text { Getz (2010), Musgrave \& Raj (2009), Lampel (2011), Lena (2011), Mintel (2010) and Smith- } \\
\text { Christensen (2009) }\end{array}$} \\
\hline
\end{tabular}

This typology improves understanding of the differing music festival formats. The festivals studied herein are portrayed in Fig 5

\begin{tabular}{|c|c|c|c|c|c|}
\hline \multicolumn{6}{|c|}{ Fig 5 Nature of festivals studied } \\
\hline $\begin{array}{l}\text { Festival } \\
\text { Region }\end{array}$ & Size & $\begin{array}{l}\text { Length } \\
\text { (Days) }\end{array}$ & Genre & $\begin{array}{l}\text { Established } \\
\text { (years) }\end{array}$ & Site \\
\hline $\begin{array}{l}\text { A } \\
\text { South-west }\end{array}$ & 8000 & 3 & $\begin{array}{l}\text { Family } \\
\text { Pop }\end{array}$ & 10 & $\begin{array}{c}\text { Urban, } \\
\text { Race course }\end{array}$ \\
\hline $\begin{array}{l}\text { B } \\
\text { Midlands }\end{array}$ & 10000 & 2 & $\begin{array}{l}\text { Cause } \\
\text { related }\end{array}$ & 36 & $\begin{array}{c}\text { Urban, } \\
\text { City park }\end{array}$ \\
\hline $\begin{array}{l}\mathbf{C} \\
\text { West Midlands }\end{array}$ & 8000 & 3 & $\begin{array}{l}\text { Dance } \\
\text { Family }\end{array}$ & 18 & $\begin{array}{l}\text { Rural, } \\
\text { Farm }\end{array}$ \\
\hline $\begin{array}{l}\text { D } \\
\text { Humberside }\end{array}$ & 5000 & 3 & $\begin{array}{l}\text { Family } \\
\text { Folk }\end{array}$ & 32 & $\begin{array}{c}\text { Urban, } \\
\text { Race Course }\end{array}$ \\
\hline $\begin{array}{l}\text { E } \\
\text { North West/ } \\
\text { Yorkshire }\end{array}$ & 300 & $3 / 2$ & Indie & 2 & $\begin{array}{c}\text { Split site: } \\
\text { Rural, Farm/ } \\
\text { Urban, Leeds } \\
\text { City venues }\end{array}$ \\
\hline $\begin{array}{l}\mathbf{F} \\
\text { Wales }\end{array}$ & 10000 & 3 & $\begin{array}{l}\text { Family } \\
\text { Pop }\end{array}$ & 5 & $\begin{array}{c}\text { Rural } \\
\text { National trust }\end{array}$ \\
\hline $\begin{array}{l}\text { G } \\
\text { North East }\end{array}$ & 8000 & 3 & $\begin{array}{l}\text { Family } \\
\text { Pop }\end{array}$ & 5 & $\begin{array}{c}\text { Rural } \\
\text { Deer Park }\end{array}$ \\
\hline
\end{tabular}

This case study conforms to social constructionism in seeking understanding of the different interpretations linking the phenomena (i.e. social considerations) and actors (i.e. the festivals' stakeholders). It is cross-sectional having been conducted in the 2014 'season' and 
focuses on 7 independent UK family-oriented music festivals with durations greater than one day (Fig 5). None of the festivals featured are overtly 'sustainable' compared to say Shambala (see http://www.shambalafestival.org). Whilst there is a reasonable geographic spread, not all regions were covered (see Fig 3). All bar one (Festival B) were private-sector events and there is a bias towards family oriented events so the insights generated herein cannot be extrapolated to other types of festival. Figs 4 and 5 do not however identify tensions amongst the stakeholders therein.

\section{A Music Festival Stakeholder typology}

The sense of participating in festivals constitute cognitive and social experiences that can induce intense feelings of belonging (Skov \& Meier, 2011). Lena (2011) warns against forgetting the variety of groups that exist including those not necessarily closely linked to the sector (Moeran \& Strandgaard-Pedersen, 2011). Hence it is useful to recognise the nature of festival stakeholders (Fig 6). 
Fig 6 Typology of music festival stakeholders

\begin{tabular}{|c|c|c|c|}
\hline Stakeholders & Specific actors & Symbol & Issues/comments \\
\hline Festivalgoers & Single, families, in groups & & $\begin{array}{l}\text { May be genre specific or } \\
\text { music industry }\end{array}$ \\
\hline $\begin{array}{l}\text { Festival } \\
\text { organisation }\end{array}$ & $\begin{array}{l}\text { Organisers } \\
\text { Volunteers } \\
\text { Directors } \\
\text { Managers } \\
\text { Officers } \\
\text { Green Leader }\end{array}$ & $\begin{array}{l}\mathrm{FO} \\
\mathrm{V} \\
\mathrm{D} \\
\mathrm{M} \\
\mathrm{O}_{f} \\
\mathrm{GL}\end{array}$ & $\begin{array}{l}\text { Employees may be legally or } \\
\text { contractually bound }\end{array}$ \\
\hline Co-producers & $\begin{array}{l}\text { Artists } \\
\text { Associations (e.g. AIF, AFO) } \\
\text { Marketers } \\
\text { Co-promoters } \\
\text { Food \& drink sales } \\
\text { Third parties } \\
\text { Charities }\end{array}$ & $M_{k}$ & \\
\hline Facilitators & $\begin{array}{l}\text { Government } \\
\text { Local \& national media } \\
\text { Sponsors }\end{array}$ & $S_{p}$ & $\begin{array}{l}\text { Parties providing cash grants } \\
\& \text { coverage; distributor \& co- } \\
\text { promoter }\end{array}$ \\
\hline $\begin{array}{l}\text { Allies/ } \\
\text { collaborators }\end{array}$ & $\begin{array}{l}\text { Professional organisations } \\
\text { Music industry } \\
\text { Trade bodies (AIF, AFO) } \\
\text { Community representatives }\end{array}$ & $\mathrm{C}_{\mathrm{r}}$ & $\begin{array}{l}\text { Those involved in other } \\
\text { festivals; in-kind support }\end{array}$ \\
\hline Regulators & $\begin{array}{l}\text { Government } \\
\text { Associations } \\
\text { Benchmarking bodies }\end{array}$ & & \\
\hline Suppliers & Profit \& non-profit making & $\mathrm{S}_{\mathrm{u}}$ & $\begin{array}{l}\text { Agencies, Production, Food, } \\
\text { Merchandise, Water } \\
\text { supplies, Utilities, Waste } \\
\text { management, Infrastructure }\end{array}$ \\
\hline $\begin{array}{l}\text { Retailers/ } \\
\text { vendors }\end{array}$ & Profit \& non-profit making & $\mathrm{R}$ & $\begin{array}{l}\text { Food, Merchandise } \\
\text { Community services }\end{array}$ \\
\hline Land-owners & $\begin{array}{l}\text { Race-course } \\
\text { Charitable trusts } \\
\text { Family } \\
\text { Farmers } \\
\text { Local government } \\
\text { Independent venues }\end{array}$ & & \\
\hline The impacted & $\begin{array}{l}\text { Charities } \\
\text { Community at large } \\
\text { Special interest groups } \\
\text { Pressure groups }\end{array}$ & & $\begin{array}{l}\text { Present \& future host } \\
\text { community }\end{array}$ \\
\hline \multicolumn{4}{|c|}{$\begin{array}{l}\text { Source: adapted from Andersson \& Getz (2009); Bird (2007); Collins (2010); Getz } \\
\text { (2010); Haven-Tang \& Jones (2009); Lampel (2011); Lena (2011); Moeran \& } \\
\text { Strandgaard-Pedersen (2011); Musgrave \& Raj (2009); Sapsed (2008) }\end{array}$} \\
\hline
\end{tabular}

This typology aids understanding of music festival stakeholders. However, Lena (2011) notes the multiple values held by festival stakeholders. So organisers may also be proprietors who fulfil a number of organizational roles: producer, agent, marketer and retailer (UNCTAD, 2008). This complexity impacts on issues germane to social considerations due to potential conflicts of interest. 
Finally, Carroll (1991) referred to the environment as a stakeholder which resonates with Walsh et al (2003) who argue human welfare includes such constructs as justice, social responsibility and environmental stewardship. Since 'green' issues are considered separately in SD, stakeholders denote people and organisations herein.

\section{Sampling}

Theoretical sampling seeks cases that are likely to replicate or extend emergent theory (Eisenhardt \& Graebner, 2007). Differing approaches were used to identify and contact organisers. The Association of Festival Organisers (AFO) provided introductions to their members. Of these Festivals A, B and D (Fig 5) consented to the research. Festival G was approached via their marketing agency, $\mathrm{E}$ via a music conference whereas $\mathrm{C}$ and $\mathrm{F}$ were approached directly using details from public domain sources. Having identified the organisers, further respondents were recruited using the snowballing technique, as had previous studies into festivals (Lena, 2011) and social considerations (Young et al, 2010). All respondents herein were adults.

This paper uses numerous, highly knowledgeable informants who view the phenomena from diverse perspectives (Eisenhardt \& Graebner, 2007). Fig 7 portrays how the respondents represent different hierarchical levels, functional areas, groups and related organisations (ibid).

\begin{tabular}{|c|c|c|c|c|c|c|c|}
\hline \multicolumn{8}{|c|}{ Fig 7 Nature of stakeholders and interviews } \\
\hline Festival & Stakeholder & Connectedness & Role & Symbol & $\begin{array}{l}\text { Interview } \\
\text { Location }\end{array}$ & $\begin{array}{l}\text { Timing } \\
\text { (mins) }\end{array}$ & $\begin{array}{l}\text { Word } \\
\text { count }\end{array}$ \\
\hline \multirow[t]{3}{*}{ A } & Marketing Manager & Agency & Manager & $M_{k} M^{A}$ & Onsite & 25 & 1872 \\
\hline & Festival Organiser & Employed & \begin{tabular}{|l|} 
Executive \\
\end{tabular} & $\mathrm{FO}^{\mathrm{A}}$ & Onsite & 32 & 4514 \\
\hline & Production Director & Subcontractor & \begin{tabular}{|l|} 
Proprietor \\
\end{tabular} & $\mathrm{PD}^{A}$ & Onsite & 24 & 3533 \\
\hline \multirow[t]{3}{*}{ B } & Retailer & & Proprietor & $\mathrm{R}^{\mathrm{B}}$ & Onsite & 27 & 2910 \\
\hline & $\begin{array}{l}\text { Festival Organiser } \\
\text { Volunteer Manager }\end{array}$ & $\begin{array}{l}\text { Volunteer } \\
\text { Volunteer }\end{array}$ & $\begin{array}{l}\text { Executive } \\
\text { Management }\end{array}$ & $\begin{array}{l}\mathrm{FO}^{\mathrm{B}} \\
\mathrm{VM}^{\mathrm{B}}\end{array}$ & Onsite & 29 & 3287 \\
\hline & $\begin{array}{l}\text { Community } \\
\text { representative/ } \\
\text { Retailer }\end{array}$ & Public & Management & $\mathrm{C}_{\mathrm{r}}^{\mathrm{B}}$ & Onsite & 26 & 3528 \\
\hline \multirow[t]{5}{*}{ C } & Supplier/retailer & Supplier & Proprietor & $\mathrm{S}_{\mathrm{u}}^{\mathrm{C}}$ & Offsite & 27 & 3522 \\
\hline & Retailer/supplier & Supplier & Management & $\mathrm{R}^{\mathrm{C}}$ & Onsite & 33 & 4695 \\
\hline & Festival Organiser & Employed & Executive & $\mathrm{FO}^{\mathrm{C}}$ & Onsite & 44 & $\mathrm{n} / \mathrm{a}$ \\
\hline & Green Leader & Volunteer & Management & $\mathrm{GL}^{\mathrm{C}}$ & Onsite & 28 & 2792 \\
\hline & Volunteer Manager & Volunteer & Management & $\mathrm{VM}^{\mathrm{C}}$ & Onsite & 10 & 1792 \\
\hline \multirow[t]{2}{*}{$\mathrm{D}$} & Marketing Manager & Employed - P/T & Management & $\mathrm{M}_{\mathrm{k}} \mathrm{M}^{\mathrm{D}}$ & Onsite & 46 & 7732 \\
\hline & Festival Organiser & Employed & Executive & $\mathrm{FO}^{\mathrm{D}}$ & Onsite & 24 & 4266 \\
\hline$E$ & Festival Organiser & Employed & Executive & $\mathrm{FO}^{\mathrm{E}}$ & Offsite & 45 & 6648 \\
\hline \multirow[t]{2}{*}{$\bar{F}$} & Marketing Officer & Supplier & Officer & $\mathrm{M}_{\mathrm{k}} \mathrm{O}_{\mathrm{f}}^{\mathrm{F}}$ & Onsite & 7 & 1126 \\
\hline & $\begin{array}{l}\text { Sponsor/ Community } \\
\text { Representative }\end{array}$ & Public & Management & $\mathrm{S}_{\mathrm{p}}^{\mathrm{r}}$ & Onsite & 15 & 2061 \\
\hline \multirow[t]{2}{*}{ G } & Production Director & Employed & Executive & $P D^{G}$ & Offsite & 49 & 6372 \\
\hline & $\begin{array}{l}\text { Festival Organiser }{ }^{\#_{1}} \\
\text { Festival Organiser }{ }_{2}\end{array}$ & $\begin{array}{l}\text { Employed } \\
\text { Employed }\end{array}$ & $\begin{array}{l}\text { Executive } \\
\text { Executive }\end{array}$ & $\begin{array}{l}\mathrm{FO}^{\mathrm{G}} \\
\mathrm{FO} 2^{\mathrm{G}}\end{array}$ & $\begin{array}{l}\text { Offsite } \\
\text { Offsite }\end{array}$ & 90 & 13521 \\
\hline
\end{tabular}


Semi-structured interviews with 21 stakeholders generated substantial qualitative data. Fig 7 also improves comprehension as when reflecting on insights, knowing a respondent is a supplier (who may be averse to criticising the organiser) is useful. Furthermore social desirability bias may exist, as respondents may want to be perceived to be more 'worthy'.

17 of the interviews took place during the 'live' festival, onsite and often in unusual environments. For example, some Festival A stakeholders were interviewed backstage often in sight of the artists e.g. the Boomtown Rats. The festival $\mathrm{C}$ organiser $\left(\mathrm{FO}^{\mathrm{C}}\right)$ was interviewed in the press tent immediately next to the Dance stage whereas the Green Leader $\left(\mathrm{GL}^{\mathrm{C}}\right)$ and Volunteer Manager $\left(\mathrm{VM}^{\mathrm{C}}\right)$, who were dressed as 'Cave People', took place on a hillside overlooking the site. The Festival $D$ organiser $\left(F O^{D}\right)$ was interviewed on a main thoroughfare as internationally acclaimed artists walked past. Hence the interviewing necessitated a degree of pragmatism as the respondents were naturally prioritising their festivals.

\section{FINDINGS AND DISCUSSION}

The research objectives shaped the topic guide used as part of the qualitative, interpretive process (Fig 8).

\begin{tabular}{|l|l|}
\hline \multicolumn{2}{|c|}{ Fig 8 Topic Guide } \\
\hline Topics & Theoretical underpinning \\
\hline Terminology & Barkemeyer (2009); Barkemeyer et al (2009; Elkington (1998, 2004); Carroll (1991) \\
\hline Respect & Garriga \& Mele (2004); Haberberg et al (2010) \\
\hline Transparency & Oates et al (2008) \\
\hline Adoption & Jones at el (2009); Dulewicz \& Higgs (2003); Tilley \& Young (2009) \\
\hline Social inclusion & Anderson (2017); Margolis \& Walsh (2003) \\
\hline Ethics & Belz \& Peattie (2012): Margolis \& Walsh (2003) \\
\hline Ownership & Planken et al (2013); Getz (2007, 2010) \\
\hline Compliance & Oates et al (2008); Haberberg et al (2010): Musgrave \& Raj (2009): Margolis \& Walsh (2003) \\
\hline
\end{tabular}

The topic guide provided the platform for the subsequent thematic analysis and structure for coding the data. It enabled identification of constructs embedded in the narratives (Fig 9). 
Fig 9 CSR Findings

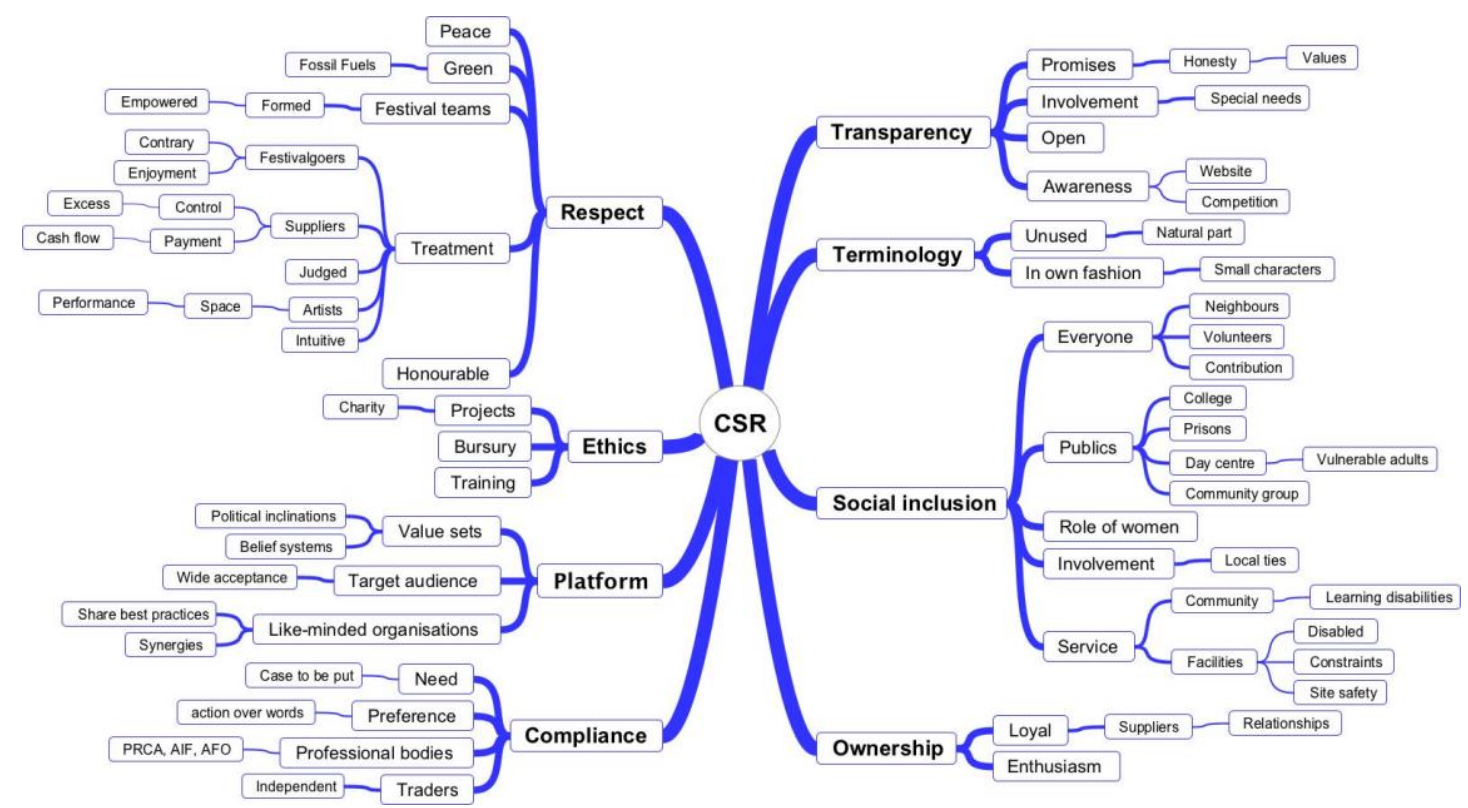

A limitation of Fig 9 is while the constructs follow the 'social considerations' literature they were neither equally weighted nor perceived universally positively. To address this it is germane to reflect on how the constructs were perceived by both stakeholder groups and to synthesise the findings with extant literature.

\section{Terminology}

'CSR' was not recognised by most suppliers. One insisted "it's something that we've never even been aware of...until, you just said Corporate Social Responsibility" $\left(R^{C}\right)$. Another asked "what do you mean?... we get training and ... a bursary for the gym..." $\left(M_{k} O_{f}{ }^{F}\right) . A$ supplier (who had worked for large organisations) recognised CSR however maintained festivals "don't talk about it. They don't put a label on it. There's a lot of people that are out there that have a corporate responsibility platform without even realising... because of their value sets, their belief systems and their political inclinations" $\left(S_{\cup}{ }^{C}\right)$. Another supplier suggested his organisation does "it in our own fashion with small characters" $\left(C_{r}^{B}\right)$. Similarly, an organiser insisted CSR had "never been used but there's no reason to not say it ... it's just a natural part of what we do" (FOA $)$.

Apropos SD a supplier suggests it means "the regenerative use of resources...it's something that happens organically in festivals ... it always has done" $\left(S_{\cup}{ }^{C}\right)$. Some stakeholders saw SD through a financial 'lens' with one referring to macro-economic difficulties for festivals noting "in a period where you've got economic constraints ... less public purse (is) available for providing events of this nature" $\left(S_{p}{ }^{F}\right)$. Some suppliers had negative connotations e.g. 
sustainability is a "buzzword" $\left(\mathrm{M}_{k} \mathrm{M}^{\mathrm{D}}\right)$ and "quite a complicated one" $\left(P D^{\mathrm{A}}\right)$. Whilst some respondents had a narrower focus on SD, others had holistic interpretations akin to TBL. One supplier suggests it represents "a whole vastness of things that people are aware of that you're trying to tap into" $\left(\mathrm{R}^{\mathrm{C}}\right)$.

Regarding SD an organiser suggested "I think, can you keep doing it?" (FO1 $\left.{ }^{G}\right)$. An organiser linked it to values suggesting it ensures "things can continue in a safe and inclusive way... It's about respect" (FO' ${ }^{C}$. This aligns with Garriga \& Mele (2003) who advocated respecting individuals' dignity. A Production Director insisted 'the primary focus has to be that it's financially sustainable. That's the bottom line ... working financially budget wise ... (and to be sustainable is) ... to be resilient, it's very easy to be hit if something goes wrong. Maybe everyone doesn't see it that way, but it's about grip. And treating contingency seriously" $\left(P D^{G}\right)$.

Some organisers questioned SD with one opining "sustainability is a strange word... almost a meaningless term ... depending on how it's applied" $\left(\mathrm{FO}^{\mathrm{G}}{ }^{\mathrm{G}}\right)$. Another questioned sustainability due to it's ambiguity exclaiming "Oh God!, that's a big question... The festival needs to be sustainable in different ways... It's a bit difficult to describe" (FO $\left.{ }^{\mathrm{D}}\right)$. Organisers should communicate their CSR or SD adoption via coherent programmes (Oates et al, 2008) with stakeholders. They should facilitate the festivalgoers' information gathering by improving awareness of products, suppliers and socio-environmental issues (ibid).

\section{Respect}

'Respect' featured highly, for the environment and society. A supplier maintained "the markets that I wanted to be in I had to feel comfortable with. And I wanted the guys who work with me to feel comfortable" $\left(\mathrm{S}_{\mathrm{u}}{ }^{\mathrm{C}}\right)$. This enlightened approach resonates with studies that recognised suppliers comply for varying reasons (Haberberg et al, 2010).

'Respect' shaped an organiser's approach. She insisted "we have to live with ourselves. I can't lie to people ... it upsets $\mathrm{me}$ " $\left(\mathrm{FO}^{\mathrm{G}}\right)$. Organisers risk not aligning their values with festivalgoers as those with, say, ethical values may simply switch festivals. This supports Garriga \& Mele (2003) who advocated providing services efficiently and fairly, respecting individuals 'dignity and rights. Furthermore, respect contributes to social well-being and a harmonic way of co-existing (ibid). This can engender ownership and supports Planken et al (2013) who found (some) ethical marketing strategies led to more positive attitudes towards companies. 


\section{Transparency}

Newer festivals may struggle to establish ethical behaviour with a supplier discussing one that "is a great little festival but the weather was awful so we didn't do too well... they'd promised there'd only be so many traders there and got extra ones in... It's promising one thing and delivering something else ... they should have told the original caterers and said 'do you want to pay a bit more?'” $\left(R^{B}\right)$. The aforementioned organiser demonstrated a lack of transparency. Whilst organisers have obligations they may also have competing needs with other stakeholders (Jones et al, 2009).

Organisers should treat vendors equitably and manage their expectations. Transparency underpinned an organiser's decision to only offer weekend tickets as "when we say that there are this many people that is the case...it is us being honest" $\left(\mathrm{FO}^{G}\right)$.

\section{Adoption}

A supplier warned "the way the larger world is going is not sustainable everybody knows that but nobody will do anything about it until it has to be done" $\left(R^{B}\right)$. As discussed, CSR was largely not recognised and no respondents had CSR platforms. Furthermore, most stakeholders do not necessarily consider themselves to be sustainable. Sustainable (particularly green) practices were undertaken intuitively with a marketer insisting organisers "don't think of it as anything special... It's so intuitive it's like 'why wouldn't you do that'?" $\left(M_{k} M^{D}\right)$. This aligns with those who believe the truly successful organizations will be those which can address the needs and interests of all stakeholders and manage the paradoxes resulting from the potentially conflicting needs of these audiences (Dulewicz \& Higgs, 2003).

Some argue festivals are at the forefront of adopting sustainability. A marketer suggests "organisations like this are leading the way ...(and she would)... rather have a company do good than talk-the-talk" $\left(\mathrm{M}_{\mathrm{k}} \mathrm{M}^{\mathrm{A}}\right)$. This supports studies that identified increasing sustainability rhetoric but little progress in addressing social problems facing society (Tilley \& Young, 2009) and social considerations being an afterthought rather than forethought (Jones et al, 2009).

\section{Social inclusion}

Social inclusion is an objective for most festivals and generated comments from organisers but little from suppliers. A supplier describes how they advocate volunteering amongst those with special needs as "there are others who are quick to say we don't have facilities" $\left(C_{r}^{B}\right)$. Inclusivity is core for some, with a local government sponsor stating it is "central to all of our 
activities... (and) ...the organisers have done their utmost to be as inclusive as possible" $\left(S_{p}{ }^{F}\right)$.

That said, social inclusion involves sacrificing profitability. One stakeholder maintained "one of our tenets is to make sure there's always... free access and we put on a lot of kids events ... that people don't pay for and that's because we want to give a service ... to the community" $\left(\mathrm{M}_{\mathrm{k}} \mathrm{M}^{\mathrm{D}}\right)$. This chimes with Margolis \& Walsh (2003) who insist organisations make social investments in the face of compelling economic counter-arguments.

One organiser insisted "it's supposed to be inclusive for everyone in ... the surrounding areas...(to)... see their neighbours and they see people that they didn't know" (FOC). Another suggests "it's a really big thing that we're trying to support and get people on board" $\left(F O^{D}\right)$. Furthermore "We do what we can to be as inclusive as possible ... where possible we put ourselves out to help people. We can charge electric scooters ... we have a viewing platform for the main stage" $\left(G L^{C}\right)$. These findings align with Andersen (2017) who argued leaders influence responsible behaviour

\section{Ethics}

A supplier insists organisers do not 'flag' (i.e. communicate) ethical behaviour as "It's widely accepted in the target audience, the festivalgoers. Without it I don't think they'd get the same target audience, the same ticket sales" $\left(S_{U}{ }^{C}\right)$. Similarly Belz \& Peattie (2012) advocate considering the customers' wants, needs and the socio-ecological problems to which they relate, in a balanced and co-ordinated way. Many suppliers cited their support for charities as evidence of their ethical behaviour. "You've got Amnesty International here, .... Some of the local ones like Martins Hospice" $\left(C_{r}^{B}\right)$. Other examples "World Vision" (ibid), "Oxfam" $\left(\mathrm{S}_{U}{ }^{\mathrm{C}}\right)$ and Action $21\left(\mathrm{C}_{\mathrm{r}}^{\mathrm{B}}\right)$ a local charity (come umbrella organisation) that supports other charities. This supports Margolis \& Walsh (2003) who found highly recognisable partners could reduce uncertainty and provide reputational benefits.

One marketer cites having "a strong community message, whether we communicate it as fully as we could is probably debatable" $\left(M_{k} M^{D}\right)$. Another marketer suggested "we're starting to work on projects where there's an element of that involved. Whether Ethical or whether it's charity? ...I know they are two different things" $\left(\mathrm{M}_{\mathrm{k}} \mathrm{M}^{\mathrm{A}}\right)$. This supports Margolis \& Walsh (2003) who argued communication represents a key challenge for those advocating CSR. 


\section{Ownership}

The stakeholders see ownership in a sophisticated manner where those involved 'take ownership' across a range of aspects. It can be a form of responsibility with one organiser describing how the incidences of lost children were reduced because "we think the parents were ringing numbers on lost children's wristbands" $\left(\mathrm{FO} 1^{\mathrm{G}}\right)$. Hence it influences the atmosphere as values such as 'safety' concerns are more likely to be addressed if parents think their peers will help care for the children onsite. This supports those who recognise consumers as co-creators in the creative industries (UNCTAD, 2008).

\section{Compliance}

Suppliers expressed diverging views regarding enforcing compliance with one warning "I've seen the trivialisation of the subject. I don't want to see necessarily the trivialisation of ... sustainability by standards that are designed to make the non-compliant compliant" $\left(\mathrm{S}_{\mathrm{u}}{ }^{\mathrm{C}}\right)$. This counters extant studies suggesting compliance needs to be adhered to and, where necessary, corrective actions should be taken (Musgrave \& Raj, 2009).

The organisers were aware (generally) of sustainable event management standards however only 2 could cite ISO 20121. One $\left(\mathrm{GL}^{\mathrm{C}}\right)$ was a sustainability officer for a Local Authority, the other a free-lance Production Director $\left(P D^{A}\right)$; he suggested the small size of most festivals is a barrier (to compliance) noting "Festival Republic may have a complete team working on just one festival ... they have the time the money and the resources (however) ...small festivals like Wychwood and Larmer Tree use independent festival producers and ... it would be impossible". Hence size is a perceived barrier to adoption for festivals and their suppliers. This resonates with studies where standards are viewed with suspicion (Oates et al, 2008).

The findings apropos the constructs are summarised in Fig 10 


\begin{tabular}{|c|c|c|c|c|c|}
\hline \multicolumn{6}{|c|}{ Fig 10 Comparative analysis of stakeholder groups' perceptions of constructs } \\
\hline & \multicolumn{2}{|c|}{ Organisers (and employees) } & \multicolumn{2}{|l|}{ Suppliers } & \multirow[t]{3}{*}{ Objective } \\
\hline & CSR & SD & CSR & SD & \\
\hline \multicolumn{5}{|l|}{ Construct } & \\
\hline $\begin{array}{l}\text { Respect } \\
\text { (Broad } \\
\text { agreement) }\end{array}$ & $\begin{array}{l}\text { Featured highly } \\
\text { for society }\end{array}$ & $\begin{array}{l}\text { Featured highly for } \\
\text { the environment } \\
\text { and society }\end{array}$ & $\begin{array}{l}\text { Featured highly } \\
\text { for society }\end{array}$ & $\begin{array}{l}\text { Featured highly } \\
\text { for the } \\
\text { environment and } \\
\text { society }\end{array}$ & $\mathrm{RO}_{1}$ \\
\hline $\begin{array}{l}\text { Adoption } \\
\text { (Broad } \\
\text { agreement) }\end{array}$ & $\begin{array}{l}\text { Leaders seek to } \\
\text { influence } \\
\text { behaviour of } \\
\text { others }\end{array}$ & $\begin{array}{l}\text { Green practices } \\
\text { undertaken; yet do } \\
\text { not see themselves } \\
\text { as sustainable }\end{array}$ & $\begin{array}{l}\text { Some support } \\
\text { for volunteering } \\
\text { and charities }\end{array}$ & $\begin{array}{l}\text { Festivals are } \\
\text { considered to be } \\
\text { at the forefront } \\
\text { of adopting } \\
\text { sustainability }\end{array}$ & $\mathrm{RO}_{2}$ \\
\hline $\begin{array}{l}\text { Social inclusion } \\
\text { (Broad } \\
\text { agreement) }\end{array}$ & $\begin{array}{l}\text { An objective } \\
\text { for most } \\
\text { festivals and } \\
\text { large sponsors }\end{array}$ & & $\begin{array}{l}\text { Not a } \\
\text { consideration for } \\
\text { small suppliers } \\
\text { and vendors. }\end{array}$ & & $\mathrm{RO}_{2}$ \\
\hline $\begin{array}{l}\text { Ethics } \\
\text { (Broad } \\
\text { agreement) }\end{array}$ & $\begin{array}{l}\text { Many festivals } \\
\text { have ethical } \\
\text { projects often } \\
\text { including } \\
\text { charities }\end{array}$ & $\begin{array}{l}\text { One organiser } \\
\text { alludes to a strong } \\
\text { community } \\
\text { message but } \\
\text { questions own } \\
\text { communication } \\
\end{array}$ & $\begin{array}{l}\text { Some think } \\
\text { festivals do not } \\
\text { 'flag' ethical } \\
\text { behaviour as it is } \\
\text { assumed }\end{array}$ & & $\mathrm{RO}_{1} / \mathrm{RO}_{2}$ \\
\hline $\begin{array}{l}\text { Transparency } \\
\text { (Divergence) }\end{array}$ & $\begin{array}{l}\text { Differs across } \\
\text { festivals; } \\
\text { organisers } \\
\text { believe they } \\
\text { treat suppliers } \\
\text { equitably }\end{array}$ & $\begin{array}{l}\text { Newer festivals } \\
\text { struggle to adopt } \\
\text { measures }\end{array}$ & $\begin{array}{l}\text { Need clarity and } \\
\text { consistency } \\
\text { from organisers }\end{array}$ & $\begin{array}{l}\text { Increasing } \\
\text { sustainability } \\
\text { rhetoric but } \\
\text { progress needed } \\
\text { in treating } \\
\text { suppliers } \\
\text { equitably across } \\
\text { sector }\end{array}$ & $\mathrm{RO}_{2}$ \\
\hline $\begin{array}{l}\text { Ownership } \\
\text { (Divergence) }\end{array}$ & $\begin{array}{l}\text { Organisers see } \\
\text { ownership } \\
\text { extending to } \\
\text { suppliers and } \\
\text { festivalgoers }\end{array}$ & $\begin{array}{l}\text { Overtly } \\
\text { sustainable } \\
\text { festivals insist on } \\
\text { suppliers adopting } \\
\text { SD approaches }\end{array}$ & $\begin{array}{l}\text { More of an issue } \\
\text { for organisers } \\
\text { than suppliers }\end{array}$ & $\begin{array}{l}\text { Suppliers have } \\
\text { differing } \\
\text { motivations and } \\
\text { may be } \\
\text { enlightened, } \\
\text { coerced or } \\
\text { mimetic }\end{array}$ & $\mathrm{RO}_{2}$ \\
\hline $\begin{array}{l}\text { Compliance } \\
\text { (Divergence) }\end{array}$ & $\begin{array}{l}\text { No CSR } \\
\text { platform or } \\
\text { official policy } \\
\text { amongst } \\
\text { festivals } \\
\text { studied }\end{array}$ & $\begin{array}{l}\text { Aware of } \\
\text { sustainable event } \\
\text { management } \\
\text { standards and } \\
\text { green } \\
\text { benchmarking } \\
\text { sites }\end{array}$ & $\begin{array}{l}\text { NO knowledge } \\
\text { of CSR } \\
\text { standards or } \\
\text { benchmarks. }\end{array}$ & $\begin{array}{l}\text { Some awareness } \\
\text { of standards; } \\
\text { wary of forced } \\
\text { compliance; } \\
\text { concerns about } \\
\text { trivialisation }\end{array}$ & $\mathrm{RO}_{3}$ \\
\hline $\begin{array}{l}\text { Terminology } \\
\text { (Divergence) }\end{array}$ & $\begin{array}{l}\text { Limited } \\
\text { awareness }\end{array}$ & $\begin{array}{l}\text { Broad awareness } \\
\text { of sustainability } \\
\text { though not TBL. } \\
\text { Agreed with the } \\
\text { principle. Some } \\
\text { definitional } \\
\text { ambiguity }\end{array}$ & $\begin{array}{l}\text { Little awareness } \\
\text { amongst the } \\
\text { sole-traders, } \\
\text { micro- } \\
\text { enterprises or } \\
\text { SMEs }\end{array}$ & $\begin{array}{l}\text { Broad awareness } \\
\text { of sustainability } \\
\text { though not TBL. } \\
\text { Agreed with the } \\
\text { principle. Some } \\
\text { definitional } \\
\text { ambiguity }\end{array}$ & $\mathrm{RO}_{1}$ \\
\hline
\end{tabular}

\section{CONCLUSIONS}

Most festivals operated between Carroll's Discretionary and Ethical levels (Fig 1) as they often exceeded stakeholders' expectations, were philanthropic and typically went beyond the basic legal requirements in order to do what is just and fair. 
As no widely acceptable typology had emerged (Getz, 2010) this study created typologies for festivals and stakeholders (Figs $4 \& 6$ ) and thus contributes to the study of festivals.

Apropos terminology, the phrase CSR lacks awareness amongst the respondents who were sole-traders, micro-enterprises or SMEs. This finding counters previous studies suggesting CSR is frequently used in businesses (Barkemeyer et al, 2009). Whilst most studies germane to social considerations relate to large organisations this paper relates to inherently small organisations. However, most respondents were aware of sustainability and largely perceived it positively.

The 'Respect', 'Social inclusion', 'Adoption' and 'Ethics' constructs reflected broad agreement between the stakeholder groups. Previously there has been little research into the transient relationships between festivals and their suppliers. Hence it is useful to recognise the synergies in these constructs. Taken together these constructs suggest social considerations are viewed positively and valued by both stakeholder groups, which satisfies $\mathrm{RO}_{1}$.

However, when considering $\mathrm{RO}_{2}$ there are divergences between the organisers and suppliers regarding the 'Transparency', 'Ownership' and 'Compliance' constructs. These divergences could impede the implementation of social considerations.

Whilst both stakeholder groups agreed in principle with the notion of transparency, divergence arose from the suppliers servicing different festivals. Organisers may be consistent within their own domain however clearly transparency differs across festivals. This may result from the number and diverse nature of music festivals (Fig 3 and 4).

Ownership is more of an issue for organisers than suppliers. Organisers must recruit many suppliers to deliver each festival. Suppliers compete with each other for the opportunity to supply the festivals and may adapt their approaches mimetically or even feel coerced rather than being enlightened (Haberberg et al, 2010).

Whilst the organisers were aware of sustainable event management standards, the suppliers were largely unaware and conflicted apropos compliance. The suppliers were wary of being forced to comply on grounds of resources (with festivals and suppliers being predominantly SMEs). One argued forced compliance could lead to the trivialisation of the subject. This counters studies suggesting compliance needs to be adhered to and, where necessary, corrective actions should be taken (Musgrave \& Raj, 2009). 


\section{PRACTICAL IMPLICATIONS}

Having reflected on the synergies and divergences in relation to specific constructs $\left(\mathrm{RO}_{2}\right)$, it is appropriate to suggest practices to address divergences between the stakeholder groups apropos the adoption of social considerations $\left(\mathrm{RO}_{3}\right)$.

Most stakeholders did not recognise the explicit terms 'Corporate Social Responsibility'. It was evident that they undertook practices that resonated with 'CSR'. However, no festivals had defined (CSR) policies and/or platforms whereas some were referring to sustainability in the task environment (i.e. onsite), on websites and on social media sites. Festivals should facilitate the festivalgoer's information gathering by improving awareness of products, suppliers and socio-environmental issues (Oates et al, 2008).

The issue of compulsory 'compliance' with standards was met with variance if not resistance. Findings suggest trust and reputations are shaped by previous experiences, behaviours and co-operative efforts. Festivals and suppliers should be encouraged to promote, and where resources are available, adopt ISO 20121. This will raise awareness of such standards and improve the motivation of both stakeholder groups to also take ownership of social considerations.

Apropos transparency, festivals must be consistent when dealing with suppliers. Interfestival consistency can only arise through collaboration. This could be facilitated by representative organisations such as the AFO and the Association of Independent Festivals (see https://aiforg.com). These bodies could provide advice or alternatively benchmarking services for social considerations. Such services already exist for environmental concerns (see https://www.juliesbicycle.com)

\section{FURTHER RESEARCH}

Further insights would be gained from comparative studies of small and large festivals. There is also scope for ascertaining whether the insights apply to other industries and sectors.

It would be insightful if longitudinal research could identify trends apropos the CSR: sustainability dichotomy. These could be triangulated with research into the attitudes of festivalgoers. 
Future studies could investigate why CSR has such low recognition amongst SMEs, entrepreneurs and micro-enterprises. This may result from the term 'Corporate' as all of the respondents were committed to social practices (e.g. promoting social inclusion).

The 'Transparency', 'Ownership' and 'Compliance' constructs suggest areas for research individually and collectively.

Finally the role of representative bodies as facilitators of social considerations would be worthy of research. 


\section{References}

Agle, B.R., Donaldson, T, Freeman, RE, Jensen, M.C., Mitchell, R.K., \& Wood, D.J. (2008) Dialogue: Toward superior stakeholder theory. Business Ethics Quarterly. 18 (2), 153-190

Andersen, T. J. (2017) Corporate responsible behavior in multinational enterprise, International Journal of Organizational Analysis, 25(3), 485-505 Andersson, T.D \& Getz, D. (2009) Tourism as a mixed industry: Differences between private, public and not-for-profit festivals. Tourism Management. 30, 847-856

Barkemeyer, R. (2009) Beyond compliance - below expectations? CSR in the context of international development._Business Ethics: A European Review. 18(3)

Barkemeyer, R., Figge, F., Holt, D. and Hahn, T. (2009) What the Papers Say: Trends in Sustainability - A Comparative Analysis of 115 Leading National Newspapers Worldwide Journal of Corporate Citizenship, 33, 69-86

Belz, F-M. \& Peattie, K. (2012) Sustainability Marketing - A global perspective. $2^{\text {nd }}$ Ed. Wiley \& sons: Chichester

Bowen, H.E. \& Daniels, M.J. (2005) Does the music matter? Motivations for attending a music festival. Event Management. 9, 155-164.

Carroll, A. (1991) The Pyramid of Corporate Social Responsibility: Toward the Moral Management of Organisational Stakeholders. Business Horizons, 1991, July-August.

Dulewicz, V. \& Higgs, M. (2003) LEADERSHIP AT THE TOP: THE NEED FOR EMOTIONAL INTELLIGENCE IN ORGANIZATIONS, The International Journal of Organizational Analysis, 11(3), 193-210

Eisenhardt, K.M. \& Graebner, M.E. (2007) Theory Building from Cases: Opportunities and challenges. The Academy of Management Journal. 50 (1) 25-32

Elkington, J. (1998) The 'Triple Bottom Line' for $21^{\text {st }}$ century Business. Published in the Earthscan Reader in Business \& Sustainable Development (2001). Editors Starkey \& Welford. Earthscan Publishing: London

Elkington, J. (2004) Enter the Triple Bottom Line as cited in Henriques, A. and Richardson, J. (eds) (2004) The Triple Bottom Line: Does it all add up? London: Earthscan.

Emery, B (2012) Sustainable Marketing. Pearson Education: Harlow

Freeman (2008) ENDING THE SO-CALLED "FRIEDMAN-FREEMAN" DEBATE article from Academy of Management 2007 Symposium printed in Agle et al (2008)

Garriga, E. \& Mele, D, (2004) Corporate Social Responsibility Theories: Mapping the Territory Journal of Business Ethics. 53, 51-71

Getz, D. (2007) Event Studies: Theory, Research and Policy Planned Events. Elsevier:Amsterdam

Getz, D. (2010) The nature and scope of festival studies. International Journal of Event Management Research. 2010, 5(1) 
Haberberg, A., Gander, J., Rieple, A., Martin-Castilla, J-I and Helm, C. (2010) Institutionalizing Sustainability: the adoption of CSR practices. Journal of Global Responsibility, 1(2), 366-381

Hart, S.L. (1997) Beyond Greening: Strategies for a Sustainable World. Harvard Business Review. Jan/Feb1997, 75(1), 66-76

Henderson, S. \& Musgrave, J. (2014) Changing audience behaviour: Festival goers and throwaway tents. International Journal of Event and Festival Management, 5 (3) 247-262

ISO (2015) ISO 20121:2012 Event Sustainability management systems -- Requirements with guidance for use. Article downloaded from http://www.iso.org/iso/home/store/catalogue_tc/catalogue_detail.htm?csnumber $=54552$ [accessed 20-11-15]

Jones, B., Bowd, R. and Tench, R. (2009) Corporate irresponsibility and corporate social responsibility: competing realities Social Responsibility Journal, 5(3), 300-310

Lena, J.C. (2011) Tradition and transformation at the Fan Fair Festival. Chapter 9 in Moeran \& Strandgaard-Pedersen (2011)

Margolis, J.D. \& Walsh, J.P. (2003) Misery Loves Companies: Rethinking Social Initiatives by Business. Administrative Science Quarterly, 48, 268-305

Maeng, H.Y., Jang, H.Y \& Li, J.M. (2016) A critical review of the motivational factors for festival attendance based on meta-analysis. Tourism Management Perspectives. 17, 1625

Mintel (2010) Music Concerts and Festivals - UK - August 2010 - Market Size and Forecast

Mintel (2012) Music Concerts and Festivals - August 2012

Mintel (2017) Music Concerts and Festivals - August 2017

Moeran, B. \& Strandgaard-Pedersen, J. (2011) Negotiating Values in the Creative Industries-Fairs, Festivals and Competitive Events. Cambridge University Press: Cambridge

Moore, S.B. \& Manring, S.L. (2009) Strategy development in Sustainability all and medium sized enterprises for Sustainability and increased value creation. Journal of Cleaner Production, 17(2), 276-282.

Munoz, P. \& Dimov, D., (2015). The call of the whole in understanding the development of sustainable ventures. Journal of Business Venturing, 30(4), 632-654.

Musgrave \& Raj (2009) Introduction to a Conceptual Framework for Sustainable Events. Chapter 1 in 'Event management and Sustainability'. Edited by Raj, R. and Musgrave, J. CABI: Oxon

Oates, C., McDonald, S., Alevizou P., Hwang, K., Young, W. \& McMorland, L. (2008) Marketing Sustainability: Use of information sources and degrees of voluntary simplicity Journal of Marketing Communications, 14 (5), 351-365

Pacheco, D.F., Dean, T.J. \& Payne, D.S., (2010). Escaping the green prison:

Entrepreneurship and the creation of opportunities for sustainable development. Journal of Business Venturing, 25(5), 464-480. 
Planken, B., Nickerson, C. \& Sahu, S. (2013) CSR across the globe: Dutch and Indian consumers' responses to CSR, International Journal of Organizational Analysis, 21(3), 357-372,

Skov, L. \& Meier, J. (2011) Configuring sustainability at fashion week. Chapter 11 in Moeran, B. \& Strandgaard Pedersen, J. (2011)

Tilley, F. \& Young, W. (2009) Sustainability Entrepreneurs - Could They Be the True Wealth Generators of the Future? Greener Management International. 55, 70-92

UNCTAD (2008) Creative Economy Report 2008- The Challenge of Assessing the Creative Economy: Towards Informed Policy-making. Downloaded from unctad.org/fr/Docs/ditc20082cer_en.pdf [accessed 03-01-13]

Walker, H. \& Preuss, L.(2008) Fostering sustainability through sourcing from small businesses: public sector perspectives. Journal of Cleaner Production 2008; doi:10.1016/j.jclepro.2008.04.014.

Walsh J.P., Weber K. and Margolis J.D. (2003) Social Issues and Management: Our Lost Cause Found. Journal of Management. 29, 859-881

Windsor, D. (2001) THE FUTURE OF CORPORATE SOCIAL RESPONSIBILITY, The International Journal of Organizational Analysis, 9(3), 225-256,

Wood (2008) CORPORATE RESPONSIBILITY AND STAKEHOLDER THEORY: CHALLENGING THE NEOCLASSICAL PARADIGM. Academy of Management 2007 Symposium printed in Agle et al (2008)

Young, W., Hwang, K., McDonald, S. \& Oates, C. (2010) Sustainable Consumption: Green Consumer Behaviour when Purchasing Products Sustainable Development. 18, 20-31 